\title{
High-resolution banding: present aspects
}

\author{
M Rønne \\ Institute of Medical Biology, Department of Anatomy and Cytology, \\ Odense University, Campusvej 55, DK-5230 Odense M, Denmark
}

(Proceedings of the 9th European Colloquium on Cytogenetics of Domestic Animals; Toulouse-Auzeville, 10-13 July 1990)

high resolution / R-banding / G-banding

\section{EVOLUTIONARY ASPECTS}

Mammalian chromosomes can be defined by three structural classes visualized as the G/Q-bands, the R-bands, and the C-bands, which are associated with different functional and biochemical attributes. This chromosomal prepattern has developed during vertebrate evolution and determines the kind and quality of banding which can be obtained from different vertebrate taxa (Holmquist, 1989). The vertebrate genome is organized in very long DNA sequences of homologue base composition called isochores (Bernardi, 1989). During evolution, mammals and birds have separated from reptiles and lower vertebrates with regard to distribution and base composition of the isochores. This difference, which is reflected in the chromosomal prepattern, indicates that isochores are evolutionary units of the vertebrate genome (Bernardi, 1989). In mammals and birds, the genome can be divided into two subgenomes, the first one, the paleogenome, has not changed during vertebrate evolution. It is composed of late replicating $\mathrm{dG}-\mathrm{dC}$-poor isochores. It contains the long interspersed repeated DNA-sequences (LINES) and the tissue-specific genes (Holmquist, 1989). It corresponds to the late replicated G-band chromatin of earlycondensating chromomeres and is subjected to lyonization during embryonic development (Holmquist, 1989). The second one, the neogenome, has changed from the ancestral early replicating $\mathrm{dG}-\mathrm{dC}$-poor isochores of late-condensating interchromomeres into the early-replicating compositionally heterogeneous $\mathrm{dG}-\mathrm{dC}$-rich isochores of late-condensating interchromomeric chromatin. This subgenome contains the short interspersed repeated DNA-sequences (SINES) and the housekeeping genes (Holmquist, 1989). It corresponds to the early replicated R-band chromatin and is not subjected to any kind of inactivation during embryonic development except for the late $\mathrm{X}$-inactivation.

\section{REPLICATION PATTERNS}

DNA in mammalian chromosomes replicates in a segmented and coordinated fashion, the $S$ phase is bimodal with a distinct reduction of DNA synthesis in 
mid S phase, giving two temporal classes of replicons (Holmquist et al, 1982). The reduction in DNA synthesis corresponds to the period when the replication complex shifts from early to late replicons (Reddy and Pardee, 1980). Clusters of early and late replicons alternate and create replication patterns (Hand, 1978). The clusters may contain up to 100 individual replicons giving a DNA content which corresponds to a chromosomal band (Hand, 1978). In premature chromosome condensation (PCC) experiments, Lau and Arrighi (1981) demonstrated that S phase PCC fragments were condensed, non-replicated, chromosomal segments separated by replicating ones. Electron microscopy examinations of chromatin have revealed alternating patterns of loops and extended fibers. Each structure accounts for about half the total mass of DNA. If these structures represent the ultimate subdivision, the mammalian genome may contain 8000 bands, each with about 410 kilobases of DNA per band (Yunis and Bahr, 1979). The number of bands demonstrated in mammalian mitotic karyotypes is, however, much lower. For G-bands it amounts to about 1700 bands per haploid set (Yunis, 1981), and the RBG patterns are now demonstrated at the 1250 band stage (Drouin et al, 1988).

\section{PREPARATION TECHNIQUES}

The substrate for chromosome preparation is a single cell suspension which contains a sufficient amount of dividing cells. The procedures involve either direct preparations from proliferating tissues or establishment of cultures with proliferating potential in vitro. However, to produce chromosomal slides suitable for induction of high-resolution banding, a series of preparational procedures must be performed in an ordered and sequential manner. The number of bands which on the average can be obtained per metaphase cell depends upon the cell type, the culture, the preparation and the banding technique employed. For high-resolution banding studies, the procedures most often involve synchronization, spindle inhibition, hypotonic treatment, fixation, production of air-dried slides, and differential staining with Giemsa or fluorescent dyes. Synchronization with S phase inhibitors provides mitotic waves at known times after block release and options for precise BUdR-substitution in one of the subgenomes. In connection with synchronization, the use of spindle inhibitors can be limited or omitted without unacceptable reduction of mitotic indices. Drouin et al (1988) questioned the reason for using spindle inhibitors in association with high-resolution banding. They observed that exposure to colcemid reduced the absolute number of mitotic cells which, after harvesting and appropriate staining, displayed more than 650 bands per haploid set. However, in an exponentially growing cell culture, interphase cells continue to enter mitosis, so the total number of metaphases with long chromosomes suitable for banding does not necessarily change (Bosman et al, 1975; Rønne, 1985), and there is at present no evidence that colcemid has a direct effect on chromosome structure. Hypotonic solution induces swelling of animal cells, but chromosome contraction and morphology are influenced by the concentration and the type of salt used. If the spindle is depolymerized due to the presence of a spindle inhibitor, the chromosomes are free to swim around after the nuclear membrane has broken down at prometaphase. To equilibrate inside and outside salt concentrations, the cell will actively absorb water and start to swell in a hypotonic medium. This treatment will also free 
the chromosomes of the ribonucleoprotein complex which surrounds them. Several different hypotonic solutions have been used in conjunction with high-resolution banding in order to increase band number, however, the $0.075 \mathrm{M} \mathrm{KCl}$ solution has, in most studies, proven sufficient for the purpose. Fixation with methanol-acetic acid denatures and precipitates proteins under acid conditions. The fixative penetrates into the cells rapidly, coagulates nuclear proteins, preserves the chromosome morphology and strips cytoplasmic proteins from the cells. It has been suggested (Summer et al, 1973) that almost all the histones are removed from the chromosomes by the fixation. However, later observations (Burkholder and Duczek, 1982) have demonstrated that a substantial part of the histones is preserved during fixation. Methanol-acetic acid fixation does enhance the effect of Giemsa-staining and appears to induce conformational changes in $\mathrm{H} 1$ (Barnett et al, 1980). According to van Duijn et al (1985), the fixation causes an irreversible denaturation of the coreand H1-histones. H1 may loose its capacity to seal the DNA coils, so in a neutral buffer the negatively charged DNA helix will be attracted by the positively charged polyarginine sequences of the core histones. This causes the DNA coils to wind around the denatured core with a smaller pitch. Such processes may explain the collapses seen in SSC-treated but unstained chromosomes (Harrison et al, 1983).

\section{POSTFIXATION STAINING}

The substrate for staining procedures is the methanol-acetic acid-fixed chromosomes prepared on clean glass slides by air-drying. Staining procedures which provide a uniform unbanded appearance of the chromosomes are normally called conventional staining. Although several dyes can be used for permanent conventional staining of chromosomes, the most often used one is the Giemsa mixture. Because of continued oxidation of the dye components, the exact composition of Giemsa cannot be determined, but two dyes only, the cationic azure $B$ and the anionic eosin $\mathrm{Y}$ are required for the deep purple staining of chromatin and chromosomes. This color cannot be obtained by using azure B or eosin Y alone. The Giemsa complex has one negative and two positive charges. The positively charged part interacts with the negatively charged DNA, while the negatively charged part of the complex may interact with the positively charged histones (van Duijn et al, 1985). Since the Giemsa complex has no specificity for any particular base in the DNA, a near-neutral Giemsa solution stains the chromosomes uniformly purple if banding methods are not employed. In methanol-acetic acid-fixed chromatin, about $20 \%$ of the DNA's potential binding sites are involved in protein interactions and therefore inaccessible to the Giemsa dye. When the fixed chromatin is prepared for postfixation GTG-banding, an additional $30 \%$ of the binding sites become inaccessible. The Giemsa complex may then compete for binding sites during the staining procedure and change chromatin structure by removing or displacing proteins in the G-band DNA, while R-band proteins are bound too tightly to the DNA to allow displacement and dye precipitation. The findings that high-mobility group I (HMG-I) is located to the G/Q-bands (Disney et al, 1989) are interesting in light of GTG-band induction. The brief trypsin digestion used in GTG-band induction may preferentially remove HMG-I and thereby provide an easy access for the Giemsa complex to the G-band DNA. Trypsin is also known to digest histones. Since H1 is 
situated outside the nucleosome core, it is more exposed to trypsin during GTGbanding than the core histones. The trypsin treatment may dissociate $\mathrm{H} 1$ from the fixed nucleosomes and thereby facilitate charge rearrangements and tight linkage between $\mathrm{dG}-\mathrm{dC}$-rich DNA and the arginine-rich core histones, which may prevent the insertion of the dye complex into the negative G-bands. After hot saline treatment of chromosome slides, the centers of the chromosomes collapse leaving a rim of material around their perimeters (Harrison et al, 1983). After Giemsa-staining, transversely swollen ridges appear in G-band-positive areas. According to van Duijn et al (1985), these observations indicate that the SSC-treatment rearranges core histones. The polyarginine-rich core has a strong affinity for the dG-dC-rich R-band DNA, which may explain the swelling differences between negative and positive G-bands. Postfixation R-band induction involves incubation of chromosome slides in hot saline. This induces substantial chromatin aggregation, probably irreversible denaturation of non-histone proteins and quantitative reduction of $\mathrm{H} 1$ (Burkholder and Duczek, 1982). Chromatin aggregation takes place in the positive R-bands, so alterations in protein-DNA interactions due to differential denaturation may explain the variation in staining intensity along the chromosomes. The extraction of proteins is minimal during $\mathrm{RH}$-banding and the bands appear to be affected by the $\mathrm{pH}$ and the temperature of the surrounding medium. By varying these factors, Gbanding will replace $\mathrm{R}$-banding or vice versa (Eiberg, 1973), indicating similarities between the mechanisms of the two banding techniques. Thus, the banding technique, the $\mathrm{pH}$ of the surrounding medium, the dye concentration and dye affinity for accessible binding sites are interrelated parameters, all of which influence the equilibrium between dye and proteins. Changes in any of these parameters will change the equilibrium and, consequently, alter chromosome morphology with regard to banding structure and chromosome swelling (Rønne et al, in preparation).

\section{PREFIXATION STAINING}

Replication patterns visualized after fluorescence plus Giemsa (FPG)-staining (Perry and Wolff, 1974) resemble postfixation-induced banding. With BUdR incorporation into carly replicated DNA, the patterns correspond to G-banding, whereas the replication patterns seen after BUdR incorporation into late replicons resemble RH-banding. The effect of FPG-staining may be due to DNA loss after UV photolysis of BUdR-substituted DNA (Webber et al, 1981), BUdR concentration, the template used (Jack et al, 1989), and preferential breakage of disulfide bonds (Buys and Stienstra, 1980). BUdR-substituted DNA binds chromosomal proteins more tightly (Burkholder and Duczek, 1982) and UV irradiation acts differently on proteins in BUdR-substituted and unsubstituted chromatids (Buys and Stienstra, 1980). Jack et al (1989) suggested that the tighter binding of proteins to BUdR-substituted DNA may increase the formation of protein-protein DNA crosslinks after UV irradiation, which may exclude the Giemsa-binding sites between the histone core and DNA in a manner similar to that described for postfixation G-banding. Distamycin A, netropsin and Hoechst 33258 are ligands with preferential non-intercalative binding to dA-dT-rich DNA. Although in vitro exposure of mammalian cells to these agents seems to have similar effects on chromosome structure, the biochemical actions of distamycin $\mathrm{A}$ and netropsin are quite different from that of Hoechst 33258 
(Krey, 1980). Combined in vitro exposure of mammalian cell cultures to BUdR and one of the three ligands in late $\mathrm{S}$ and $\mathrm{G}_{2}$ phases results in BUdR substitution of late replicated DNA and a corresponding pattern of decreased chromosome contraction which can be observed after both traditional and modified Giemsa-staining (Goyanes and Mendez, 1981; Rønne, 1985). After FPG-staining, the BUdR-substituted segments appear light, the unsubstituted segments appear dark. The decreased contraction in the substituted segments enhances band resolution and band contrast (Rønne, 1985). The consequences of in vitro exposure to distamycin A, netropsin and Hoechst 33258 may not be explained only by their interactions with DNA, but also by the presence of HMG-I in the G/Q-band chromatin. According to Disney et al (1989), it is likely that the $\mathrm{dA}-\mathrm{dT}$-binding ligands in question compete with HMG-I for binding sites in the negative R-bands with inhibition of chromosome contraction and reduced accessibility for the Giemsa complex to potential dye-binding sites as a consequence. The high affinity for BUdR-substituted DNA displayed by these ligands and the FPG-staining subsequently employed in the RBG-procedure then enhances the differences between $R$ - and $G / Q$-band regions.

\section{PRESENT ASPECTS}

The high-resolution banding techniques provide the possibilities to detect chromosome breaks and rearrangements even within major bands. The developments in culture and banding techniques have been extremely fast; ten years after the Paris conference in 1971, Yunis (1981) published the haploid human karyotype at the 1700 -band stage; the number of described bands had increased by about a factor of 5 . For other mammals, the development was slower, but recently good results have narrowed the gap between human and animal cytogenetics. The increase of bands in a given karyotype is, however, not an aim by itself, but a research tool, so the value of a high band level is reduced if band quality is sacrificed in the process. When high-resolution banding is combined with other chromosomal techniques, specific sites on the chromosomes can be detected and precisely localized. High-resolution banding techniques have been extremely valuable, especially for the localization of single-copy genes and specific breakpoints. Banding based on BUdR-substitution in one of the subgenomes followed by FPG-staining is highly resistant to the hybridization procedures (Zabel et al, 1983), and the uniform mitotic population of lymphocytes prepared for subsequent RBG-banding after double synchronization (Rønne, 1985), seems to be an excellent substrate for in situ hybridization of single-copy genes (Rønne et al, 1990). Inhibitors of dihydrofolate reductase and thymidylate synthetase have been used to unmask fragile sites (Tommerup et al, 1981). In the concentration range from $10^{-7}$ to $10^{-6} \mathrm{M}$, methotrexate, 5-fluoro- $2^{\prime}$-deoxyuridine and fluorouracil also act as reversible $\mathrm{S}$ phase inhibitors, so with the traditional synchronization techniques some fragile sites may be unmasked as a side effect of the culture and banding procedures. The banding patterns obtained after differential staining of chromosomes are not only a result of the method employed, but also a consequence of isochore organization and chromosomal prepattern. The marked differences in banding potential between different cell systems obtained from the same individuals indicate, however, that other factors influence chromosome mor- 
phology and banding potential, but the exact molecular mechanisms remain to be identified.

\section{REFERENCES}

Barnett RI, Gray VA, Mackinnon EA (1980) Effects of acetic acid-alcohol, histone 1 and histone fragments on Giemsa staining patterns in chromosomes. Histochemistry 65, 207215

Bernardi G (1989) The isochore organization of the human genome. Annu Rev Genet 23, 637-661

Bosman FT, van der Ploeg M, Schaberg A (1975) Chromosome preparations of human blood lymphocytes - evaluation of techniques. Genetica $45,425-433$

Burkholder GD, Duczek LL (1982) The effect of chromosome-banding techniques on proteins of isolated chromosomes. Chromosoma 87, 425-435

Buys CHCM, Stienstra S (1980) Involvement of sulfhydryl groups of chromosomal proteins in sister chromatid differentiation. Chromosoma (Berlin) 77, 325-332

Disney JE, Johnson KR, Magnuson NS, Sylvester SR, Reeves R (1989) High-mobility group protein HMG-I localizes to G/Q and C-bands of human and mouse chromosomes. J Cell Biol 109, 1975-1982

Drouin R, Lemieux N, Richer CL (1988) High resolution R-banding at the 1250-band level. 1. Technical considerations on cell synchronization and R-banding (RHG and RBG). Cytobios 56, 107-125

Eiberg $H$ (1973) G, R and C banding patterns of human chromosomes produced by heat treatment in organic and inorganic solutions. Clin Genet 4, 556-562

Goyanes VJ, Mendez J (1981) Karyotyping chromosomes by electron microscopy. Condensation-inhibition of $\mathrm{G}$ bands in human and Chinese hamster chromosomes by a BrdU-Hoechst 33258 treatment. Cancer Genet Cytogenet 4, 45-51

Hand R (1978) Eukaryotic DNA: organization of the genome for replication. Cell 15, $317-325$

Harrison CJ, Allen TD, Harris R (1983) Scanning electron microscopy of variations in human metaphase chromosome structure revealed by Giemsa-banding. Cytogenet Cell Genet 35, 21-27

Holmquist GP (1989) Evolution of chromosome bands: molecular ecology of noncoding DNA. J Mol Evol 28, 469-486

Holmquist G, Gray M, Porter TH, Jordan S (1982) Characterization of Giemsa dark-andlight band DNA. Cell 31, 121-129

Jack EM, Harrison DJ, White GRM, Ockey CH, Allen TD (1989) Fine-structural aspects of bromodeoxyuridine incorporation in sister chromatid differentiation and replication banding. J Cell Sci 94, 287-297

Krey AK (1980) Non-intercalative binding to DNA. Prog Mol Subcell Biol 7, 43-87 Lau YF, Arrighi FE (1981) Studies of mammalian chromosome replication. II. Evidence for the existence of defined chromosome replicational units. Chromosoma 83, 721-741

Perry P, Wolff S (1974) New Giemsa method for the differential staining of sister chromatids. Nature 251, 156-158

Reddy GPV, Pardee AB (1980) Multienzyme complex for channeling in mammalian DNA replication. Proc Natl Acad Sci USA 77, 3312-3316

Rønne M (1985) Double synchronization of human lymphocyte cultures: selection for highresolution banded metaphases in the first and second division. Cytogenet Cell Genet 39, 292-295 
Rønne M, Kirpekar F, Shibasaki Y, Poulsen BS, Kristiansen K (1990) R-banding and in situ hybridization. Localization of single copy genes on high resolution banded chromosomes. Anticancer Res 10, 375-378

Sumner AT, Evans HJ, Buckland RD (1973) Mechanisms involved in the banding of chromosomes with quinacrine and Giemsa. I. The effects of fixation in methanol-acetic acid. Exp Cell Res 81, 214-222

Tommerup N, Poulsen H, Brøndum-Nielsen K (1981) 5-Fluoro- $2^{\prime}$-deoxyuridine induction of the fragile site on Xq28 associated with X-linked mental retardation. J Med Genet 18, 374-376

van Duijn R, van Prooijen-Knegt AC, van der Ploeg M (1985) The involvement of nucleosomes in Giemsa-staining of chromosomes. A new hypothesis on the banding mechanism. Histochemistry 82, 363-376

Weber LM, Brasch JM, Smyth DR (1981) DNA extraction during Giemsa differentiation of chromatids singly and doubly substituted with BUdR. Chromosoma 81, 691-700

Yunis JJ (1981) Mid-prophase human chromosomes. The attainment of 2000 bands. Hum Genet 56, 293-298

Yunis JJ, Bahr GF (1979) Chromatin fiber organization of human interphase and prophase chromosomes. Exp Cell Res 122, 63-72

Zabel BU, Naylor SL, Sakaguchi AY, Bell GI, Shows TB (1983) High-resolution chromosomal localization of human genes of amylase, proopiomelanocortin, somatostatin, and a DNA fragment (S3S1) by in situ hybridization. Proc Natl Acad Sci USA 80, 6932-6936 